\title{
FACTEURS DE RISQUE DE DÉMENCE DANS UNE POPULATION DE PERSONNES ÂGÉES SÉNÉGALAISES
}

\section{RISK FACTORS FOR DEMENTIA IN A SENEGALESE ELDERLY POPULATION}

TOURÉ Kamadore ${ }^{1}$

COUMÉ Mamadou ${ }^{2}$

NDIAYE/NDONGO NDeye Dialé ${ }^{3}$

THIAM Mamadou $\mathrm{Habib}^{3}$

ZUNZUNEGUI Maria Victoria ${ }^{4}$

BACHER Yves ${ }^{5}$

TAL/DIA Anta ${ }^{1}$

GUEYE Lamine ${ }^{6}$

SENE-DIOUF Fatou ${ }^{6}$

NDIAYE Moustapha ${ }^{6}$

THIAM Alé ${ }^{6}$

GALLO Diop Amadou ${ }^{6}$

NDIAYE Mouhamadou Mansour ${ }^{6}$

1. Département de Médecine Préventive et Santé Publique, Faculté de Médecine, Pharmacie et Odontostomatologie, Université HFaCheikh Anta Diop, Dakar-Sénégal

1. Service de Médecine Interne, CHU de HALD, Dakar-Sénégal

2. Service de Psychiatrie, CHU de Fann, Dakar-Sénégal

3. Département de Médecine Sociale et Préventive, Faculté de Médecine, Université de Montréal, Québec-Canada

4. Clinique de Mémoire, Division de Gériatrie, Sir Mortimer B Davis Hôpital Général Juif, Faculté de Médecine, Université McGill, Québec-Canada

5. Clinique Neurologique, Centre Hospitalier Universitaire de Fann, Dakar-Sénégal

E-Mail Contact - TOURÉ Kamadore : tourekamadore (at) yahoo (dot) ca

Mots-clés: Démence. Facteurs de risque. Personnes âgées. Sénégal

Keywords: Dementia. Risk factors. Elderly persons. Senegal

\section{RESUME}

\section{Description}

La démence est devenue un problème de santé publique. Dans le but d'une prévention, il est important de connaitre son épidémiologie au Sénégal. L'objectif de cette étude était d'identifier les facteurs de risque de démence dans une population de personnes âgées sénégalaises.

\section{Méthodes}

Une étude transversale a été réalisée du 01 Mars 2004 au 31 Décembre 2005 auprès d'une population de 872 personnes âgées de 55ans et plus utilisant le Centre Médicosocial et Universitaire de I'Institut de Prévoyance Retraite du Sénégal pour des soins. Par une étude en deux phases, des données sociodémographiques, sur le mode de vie, le réseau social, les antécédents ont été collectées à l'aide d'un questionnaire structuré complété par un examen clinique et une évaluation neuropsychologique. Le diagnostic de démence reposait sur des critères DSM IV-R. 


\begin{abstract}
Résultats
Cinquante huit patients $(6,6 \%)$ ont présenté la démence. Ils étaient âgés en moyenne de 67,2 ans $(( \pm 7.5)$, en majorité de sexe masculin, mariés et non-éduqués. Les antécédents médicaux les plus retrouvés étaient l'hypertension artérielle, les affections rhumatismales, respiratoires, urinaires et les troubles digestifs. Le tabagisme et la consommation d'alcool étaient rares alors que la marche était fréquente. Les personnes âgées avaient un important réseau social. L'âge, le peu de contact hebdomadaire avec les proches, les accidents vasculaires cérébraux, l'épilepsie et l'histoire familiale de démence étaient associés négativement avec la démence alors que la maladie de Parkinson et les troubles digestifs avaient un effet protecteur.
\end{abstract}

\title{
Conclusion
}

Les facteurs de risque associés à la démence dans cette population de personnes âgées Sénégalaises sont similaires à ceux observés dans les pays développés. L'intégration sociale doit occuper une place de choix pour le maintien de la fonction cognitive de la personne âgée dans cette société collectiviste

\section{SUMMARY}

\section{Background}

Aging is associated with the development of dementia. Risk factors for the disease have been elucidated in developed countries. Thus, we conducted a study to identify the risk factors for dementia in a Senegalese elderly population.

\section{Methods}

The study was cross-sectional and intended, through a two-wave process of data collection, to collect data from March 012004 to December 312005 among 872 Senegalese elderly population utilizing the Medicosocial and University Center of IPRES for health care. Sociodemographic, toxic habits, physical activity, social network, medical history, familial history of dementia data were collected with a structured questionnaire completed with a clinical exam and neuropsychological testing. Univariate, bivariate and multivariate analysis were done.

\section{Results}

Fifty eight patients $(6.6 \%)$ had dementia. The whole population has a mean age of 67.2 years $( \pm 7.5)$, mostly male, married, and non-educated. Hypertension, arthritis, gastro-intestinal diseases, respiratory diseases and genitor-urinary diseases were the main health conditions reported. Smoking and alcohol consumption were rare but walking was the main physical activity. The elderly population had a high diversity of ties and frequency of contact with the relatives and friends. Age, frequency of contact with relatives and friends, stroke, epilepsy, family history of dementia were negatively associated with dementia while Parkinson and gastro-intestinal diseases were protective.

\section{Conclusion}

The risk factors identified are similar to what exist in developed countries. Therefore, it is necessary to sensitize the health personnel, decisions-makers and the community on the existence of dementia, the risk factors to settle prevention program.

\section{INTRODUCTION}

Dans le monde, on estime qu'en 2000, le nombre de personnes démentes était de 25,5 millions représentant $0,4 \%$ de la population mondiale. Le nombre de déments va atteindre 63 millions en 2030 et 114 millions en 2050. Environ $52 \%$ vivent dans les pays sous-développés et l'augmentation du nombre de cas de démence y sera plus importante passant de 13,3 millions en 2000 à 84 millions en 2050 [60].

La démence est devenue un véritable problème de santé publique. Sa prévalence augmente considérablement avec l'âge [14]. Les autres facteurs de risque incluent: l'instruction [2], l'hérédité [28], les conditions de vie défavorisantes pendant l'enfance [10], le tabac [46] et l'alcool [13], la sédentarité [47], le faible réseau social [63] mais aussi les affections chroniques cardiovasculaires [55], respiratoires [29], l'anémie [5], le diabète [55], la maladie de Parkinson [1] et le traumatisme crânien [51] entre autres.

La population sénégalaise est estimée à 9, 526648 habitants en 2000 et elle atteindra 13 millions en 2015. 
Environ $8 \%$ de la population est âgé de 55 ans et plus et ce taux sera de $11 \%$ en 2015 . Le corollaire sera une augmentation du nombre de personnes atteintes de démence $[26,27]$.

Depuis 2004, une recherche est menée sur la démence dans la population de personnes âgées Sénégalaises. Deux hypothèses ont fondé cette recherche: 1) la prévalence de la démence sera plus faible du fait de l'organisation sociale collectiviste de la société sénégalaise avec un rôle central occupé par la personne âgée; 2) les personnes âgées avec un faible réseau social auront plus de risque de développer la démence.

L'objectif de ce présent travail était de d'identifier les facteurs de risque associés à la survenue de cette maladie dans une population de personnes âgées utilisant le Centre Médico-social et Universitaire (CMSU) de l'Institut de Prévoyance Retraite du Sénégal (IPRES) de Dakar, Sénégal pour des soins.

\section{MATERIEL ET METHODES}

\section{Site de l'étude. Le Centre Médico-social et Universitaire de l'IPRES}

L'Institution de Prévoyance Retraites du Sénégal (IPRES) gère le régime national obligatoire d'assurance vieillesse intégré au régime de sécurité sociale. Elle assure pour les titulaires de pension et leurs membres de famille, la prise en charge des frais hospitaliers et des soins de base dans ses structures sanitaires.

Le Centre Médico-Social et Universitaire (CMSU) est un centre de premiers soins assurant des consultations de gériatrie, médecine générale, gynéco-obstétrique et planification familiale, pédiatrie, ophtalmologie, odontostomatologie, rééducation fonctionnelle, urologie entre autres. Y sont associés des soins infirmiers et des examens de laboratoire (biologie et biochimie). Ces activités sont gratuites pour la personne retraitée de I'IPRES et sa famille. Les médicaments sont aussi dispensés gratuitement si disponibles au niveau de la pharmacie. Le personnel est composé de 15 agents permanents avec en plus des médecins vacataires pour améliorer la qualité des soins. Environ 6000 patients sont consultés chaque année au niveau du CMSU.

\section{Population à l'étude}

Elle était composée de personnes âgées sénégalaises 55 ans et plus qui venaient consulter au CMSU de I'IPRES pour un problème de santé et donc bénéficiaires des services offerts au niveau de cette structure de santé. Ont été exclues toutes les personnes âgées de moins de 55 ans, et celles présentant une maladie empêchant l'administration de l'instrument de collecte de données: coma, délire, aphasie, psychose, baisse importante de l'acuité visuelle et auditive.

\section{Type d'étude}

Cette étude était de type transversal avec une collecte de données en deux phases. Dans la première phase (du 01 mars 2004 au 31 décembre 2005), 872 personnes âgées de 55 ans et plus ont été interviewées. Dans la deuxième phase, celles suspectes de troubles cognitifs associées à un nombre égal de sujets normaux ont été référés pour un examen clinique complet et une évaluation neuropsychologique.

\section{Considérations éthiques}

Cette recherche a reçu l'approbation du comité d'éthique de l'Université de Montréal (Québec, Canada) et de celui du Ministère de la Santé et de la Prévention Médicale du Sénégal avant son démarrage. Un consentement éclairé avec la personne âgée ou son accompagnant était obtenu.

\section{Collecte des données \\ Le questionnaire "Vieillir au Sénégal}

C'est un instrument a été utilisé lors de la première phase pour l'interview des personnes âgées qui ont accepté de participer à l'étude. II a permis de recueillir des informations en rapport avec leurs caractéristiques sociodémographiques, leurs antécédents médicaux, une histoire familiale de démence, leur mode de vie (habitudes toxiques, activités physiques), leur réseau social complétée par une évaluation de la capacité fonctionnelle avec l'échelle adaptée de Fillenbaum $[15,37]$ et une évaluation neuropsychologique avec le Test adapté et modifié de Hodkinson [25,32] et le Test du Sénégal [56].

Le guide d'examen clinique

Il a été utilisé lors de la deuxième phase de l'étude et comprend 5 parties : 1) une revue de l'histoire de la maladie du patient ; 2) une revue des antécédents médico-chirurgicaux du patient, une éventuelle exposition à des produits toxiques, une histoire familiale de démence; 3 ) une évaluation des capacités fonctionnelles du patient [15,37]; 4) une évaluation neuropsychologique des fonctions cognitives dont la mémoire, le langage, la praxie, l'attention avec le test de l'empan, le calcul mental, l'organisation visuo-spatiale, le raisonnement avec les tests de similarité et différence, le jugement et la version nigériane adaptée du Mini Mental State Examination $[6,16] ; 5)$ un résumé de l'examen général et physique du patient incluant tous les organes; 6 ) 
l'échelle de dépression avec l'échelle "Center for Epidemiological Study of Depression (CES-D) [48]". Déroulement de la recherche

Tout patient qui a accepté de participer à cette étude a été d'abord interviewé avec le questionnaire "Vieillir au Sénégal" par un des 4 étudiants en médecine qui ont été formés au préalable pour cette tâche. Ainsi, tout patient ayant obtenu un score inférieur ou égal à 5 au Test de Hodkinson lors de l'interview était référé au chercheur principal pour un examen clinique et une évaluation neuropsychologique plus poussée avec le guide d'examen clinique. Un sujet normal apparié par le sexe était aussi référé pour comparaison. L'examen clinique était toujours précédé d'un entretien avec l'accompagnant de la personne âgée pour s'enquérir de l'histoire de la maladie, des antécédents et de l'évaluation de ses capacités fonctionnelles. Puis, nous procédions à l'examen physique complet du patient et de son évaluation neuropsychologique. En cas de suspicion de dépression, une auto-évaluation avec le CES-D était appliquée. A la fin de l'examen, l'accompagnant et le patient étaient informés des résultats de l'examen clinique. Si nécessaire, des examens complémentaires (radiographie [standard, CT scanner du cerveau], biologie, biochimie) étaient demandés et un traitement d'éventuelles affections intercurrentes institué.

Les variables à l'étude

Le diagnostic de démence a été défini selon les critères de DSM-IVR [3]. Nous avons aussi étudié d'autres variables en rapport avec:

- les caractéristiques sociodémographiques avec l'âge (en 7 catégories [55-59 ans, 60-64 ans, 65-69 ans, 70-74 ans, 75-79 ans, 80-84 ans, 85 ans et plus], le sexe (masculin, féminin), le statut matrimonial (marié(e), non-marié), l'éducation (oui, non);

- le mode de vie avec :

- les habitudes toxiques: le fait d'avoir fumé le tabac (oui, non), d'avoir consommé de l'alcool (oui, non);

- l'exercice physique : marche (oui, non); autres activités physiques: faire du vélo (oui, non), de la danse (oui, non), du jardinage (oui, non), des travaux champêtres (oui, non), de la pêche (oui, non), cette variable ayant été obtenue en faisant leur somme (score 0-5) et catégorisée en 3 modalités : "absence d'activité, 1 activité, 2 activités et plus";

- le réseau social: vit seul ou en famille (oui, non), avoir des enfants (oui, non), des frères/sœurs (oui, non), ami(e)s (oui, non), fréquence hebdomadaire des contacts avec les enfants (jamais, moins d'une fois par semaine, plus d'une fois par semaine), frères/sœurs (jamais, moins d'une fois par semaine, plus d'une fois par semaine), ami(e)s (jamais, moins d'une fois par semaine, plus d'une fois par semaine), être membre d'association communautaire (oui, non), être membre d'association religieuse (oui, non). Nous avons créée deux index: la diversité des liens avec les proches et la fréquence des contacts hebdomadaires avec les proches. La diversité des liens avec les proches est un index qui regroupe 4 items avec un score de 0 à 1 : "statut matrimonial ( $1=$ marié, $0=$ non-marié), avoir des enfants $(1=$ oui, $0=n o n)$, avoir des frères/sœurs (1=oui, $0=$ non), avoir des ami(e)s ( $1=$ oui, $0=$ non)". Pour les analyses statistiques, le score ainsi obtenu a permis de catégoriser la diversité des liens avec les proches en 3 modalités : "0-2 liens= faible, 3 liens= moyen, 4 liens= élevé". Quant à la fréquence hebdomadaires des contacts avec les proches, le score obtenu est la somme de celui obtenu avec les variables " fréquence des contacts hebdomadaires avec les enfants $(0=$ jamais, $1=$ moins d'une fois par semaine, $2=$ plus d'une fois par semaine $)$ ", " fréquence des contacts hebdomadaires avec les frères/sœurs $(0=$ jamais, $1=$ moins d'une fois par semaine, $2=$ plus d'une fois par semaine)", " fréquence des contacts hebdomadaires avec les amis ( $0=$ jamais, $1=$ moins d'une fois par semaine, $2=$ plus d'une fois par semaine) ". Elle est catégorisée en 4 modalités: "0-3 contacts, 4 contacts, 5 contacts, 6 contacts";

- des antécédents médicaux: hypertension artérielle, cardiopathie, maladies vasculaires périphériques, accident vasculaire cérébral [AVC], diabète, maladies respiratoires, maladies rhumatismales, cancer/tumeur bénigne, maladie de Parkinson, épilepsie, affections génito-urinaires, cataracte, glaucome, troubles auditifs, troubles digestifs, anémie, maladie thyroïdienne, fracture, traumatisme crânien. Toutes ces variables ont été dichotomisées "oui, non";

- I'histoire familiale de démence (oui, non).

\section{ANALYSE STATISTIQUE}

Les données recueillies ont été analysées à l'aide du logiciel SPSS- version 13.0 pour Windows. Des analyses univariées pour le calcul des fréquences, des moyennes, des écarts-types ont été effectuées. Puis, par des analyses bivariées, nous avons comparé la prévalence de la démence en fonction des caractéristiques sociodémographiques. Le test du X2 a été utilisé et les résultats exprimés avec un risque d'erreur de $5 \%$. Pour les analyses de régression logistique multivariée, nous avons déterminé l'association entre les variables indépendantes (caractéristiques sociodémographiques, habitudes de vie, réseau social, 
antécédents médicaux) et la variable dépendante (démence). Ainsi, nous avons créé un modèle en introduisant d'abord les variables sociodémographiques, ensuite les variables habitudes de vie et le réseau social et enfin les variables antécédents médicaux et l'histoire familiale de démence selon l'ordre chronologique. Ces analyses multivariées ont permis de mesurer l'association entre ces facteurs de risque et l'existence de démence avec calcul des odds ratios pour les variables qui lui sont associées. Les résultats ont été exprimés avec un intervalle de confiance à $95 \%$.

\section{RESULTATS}

Huit cent soixante douze patients âgés de 55 à 90 ans avec une moyenne de 67,2 ans $( \pm 7,5)$ ont été reçus et interviewés. Le tableau I décrit les caractéristiques sociodémographiques, le mode de vie et le réseau social de la population à l'étude. Les patients étaient du sexe masculin, marié(e)s et non-éduqués. La consommation de tabac et d'alcool était faible. La marche était pratiquée par $95,5 \%$ de la population et $84,6 \%$ ne faisaient aucune autre activité physique.

Le réseau social était caractérisé par une intense diversité des liens avec les proches et une fréquence élevée de contacts hebdomadaires avec les proches. Cependant, seuls $13,5 \%$ et $39,2 \%$ des patients étaient membre d'association communautaire et religieuse respectivement (Tableau II). L'hypertension artérielle, les affections rhumatismales, les troubles digestifs, les affections respiratoires et génito-urinaires étaient les principaux antécédents médicaux rapportés par la population de patients (Tableau III). Cinquante huit patients ont présenté une démence soit une prévalence de 6,6\%. Lors de l'analyse bivariée, la démence était significativement associée avec l'âge, l'éducation, la marche, la diversité des liens avec les proches, la fréquence des contacts avec les proches, l'hypertension artérielle, la cardiopathie, l'AVC, les affections rhumatismales, l'épilepsie, les troubles digestifs, le traumatisme crânien et l'histoire familiale de démence (Tableau III et IV). Les résultats de l'analyse de régression logistique ont permis d'identifier les principaux facteurs de risque associés à la démence. Ainsi, l'âge, la fréquence des contacts hebdomadaires avec les proches, les antécédents d'accident vasculaire cérébral, d'épilepsie et l'histoire familiale étaient indépendamment associés à la survenue de la démence dans la population à l'étude. Cependant, les antécédents de maladie de Parkinson et de troubles digestifs avaient un effet protecteur (Tableau $\mathrm{V}$ ).

\section{DISCUSSION}

Une prévalence de $6,6 \%$ pour la démence a été observée dans la population à l'étude avec comme facteurs de risque l'âge, la fréquence des contacts hebdomadaires avec les proches, les antécédents d'accident vasculaire cérébral, de maladie de parkinson, d'épilepsie, de troubles digestifs et l'histoire familiale de démence. Le rôle de l'âge dans la survenue de la démence chez la personne âgée a fait l'objet de plusieurs études au niveau clinique et populationnel. II constitue un véritable facteur de risque de survenue de cette affection. En effet, en Europe [36], aux USA [14] mais aussi en Inde [50], Corée du Sud [61], Chine [24], Brésil [23], Japon [42], Espagne [2], Israël [33] et au Nigeria [20], la démence était significativement associée à l'âge de la personne.

La personne âgée africaine occupe une place éminente dans une société où avancer en âge équivaut à gagner en dignité. Elle vivait en famille sous le même toit que ses enfants, belles-filles et petits-enfants surtout en milieu rural où cette structuration est de règle. Elle occupait une place privilégiée la plaçant au sommet de la hiérarchie sociale car gage de sécurité et gardien des valeurs traditionnelles. Malgré son âge souvent avancé, elle s'investissait dans des activités de développement de la famille et de la communauté. En plus, en tant que détentrice de secret et de sagesse, elle était souvent membre d'associations communautaires et religieuses. Cependant, avec la modernisation de la société, cette organisation sociale a tendance à disparaitre dans les villes. Ainsi, la personne retraitée âgée de surcroit perdant son pouvoir économique et social se retrouvera "abandonnée" et "dévalorisée" avec un impact sur sa santé. Dans notre population à l'étude, plus de $30 \%$ de notre population à l'étude avaient des liens faibles à modérés avec les proches et $10,2 \%$ avaient peu de contacts hebdomadaires avec eux. Le faible réseau social surtout la faible fréquentation des proches était associée à la survenue de la démence. Ce résultat vient confirmer ce qui a été observé lors d'études réalisées dans des sociétés occidentales mettant en exergue le rôle du faible réseau social dans la survenue de démence $[7,8,17,52,58,63]$. Cependant, le fait d'être membre d'association communautaire et religieuse n'était pas associé à la survenue de la démence. De nombreuses théories ont été avancées dans la physiopathologie de l'association entre le faible réseau social et la survenue de la démence chez la personne âgée: l'hypothèse de la réserve cognitive, celle vasculaire et celle liée au stress avec la 'cascade glucocorticoïde'. En effet, pour certains auteurs, le développement du réseau social entraine une plasticité neuronale avec comme conséquence une augmentation de la capacité de 
réserve du cerveau protégeant ainsi contre la détérioration cognitive [54,57]. Pour d'autres, le réseau social agit de manière indirecte via ses effets bénéfiques sur la prévention des maladies cardiovasculaires, facteurs de risque de détérioration cognitive [22]. Enfin, le faible réseau social peut entrainer un phénomène de stress qui agit sur l'axe hypothalamo-hypophyso-surrénalien avec une augmentation de la sécrétion de glucocorticoïdes. Malheureusement, cette hormone va endommager les cellules nerveuses hippocampiques avec comme conséquence une détérioration de la mémoire [41]. Bennett et al. [9] ont suivi 89 personnes âgées non-démentes et pratiqué des autopsies chez celles décédées pour étudier l'association entre le réseau social et les lésions anatomopathologiques observées au niveau du cerveau. Ils ont montré que l'importance du réseau social était associée à la gravité des lésions anatomopathologiques observées au niveau du cerveau surtout en ce qui concerne le nombre de débris neurofibrillaires. Ce résultat confirme l'importance du réseau social dans la détérioration cognitive de la personne âgée. Cependant, quel que soit le mécanisme causal, il y'a lieu de prendre en considération le rôle d'un important réseau social dans la prévention de la détérioration cognitive chez la personne âgée en général et sénégalaise en particulier face à ce bouleversement social dans lequel vit la personne âgée.

L'accident vasculaire cérébral constitue un véritable problème de santé du fait de sa prévalence élevée mais aussi en tant que l'une des premières causes de mortalité, de handicap et de démence dans le monde [59]. Au Sénégal, il représente la première cause de morbidité et de mortalité à la Clinique Neurologique du Centre Hospitalier Universitaire de Fann [45]. Dans la population à l'étude, seuls 4,8\% des patients ont présenté un AVC dans leurs antécédents et cette affection était fortement associée à la démence. La prévalence de la démence chez les survivants d'AVC est d'environ $30 \%$ et son incidence varie de $7 \%$ après la première année à $48 \%$ après 25 ans [38]. La survenue d'un AVC multiplie le risque de démence dans la population, hypothèse confirmée par des études réalisées aux USA [30], en Suède [62] et en Angleterre [43]. L'épilepsie de la personne âgée est fréquente avec une prévalence estimée à $1 \%$ après 60 ans [21] et une incidence qui augmente avec l'âge [4]. Elle relève le plus souvent d'une pathologie cérébrovasculaire, métabolique ou d'une démence. Seul $1,4 \%$ des patients de notre population à l'étude ont présenté dans leur antécédent de l'épilepsie qui était fortement associée à la démence. En effet, le rôle de l'épilepsie sur la survenue de troubles cognitifs a été bien élucidé par Griffith et al. [18] et Martin et al. [40]. D'autres études épidémiologiques ont confirmé le risque de démence chez les personnes âgées présentant des crises d'épilepsie [11]. Cependant, des questions demeurent quant aux facteurs explicatifs de cette association qui pourrait être en rapport avec la médication antiépileptique, la durée d'évolution des crises épileptiques ou la cause de l'épilepsie elle-même [18].

Quant au rôle de l'hérédité dans la transmission de la démence dans la population de personnes âgées, les résultats observés à travers notre étude confirment cette hypothèse. En effet, Hall et al. [20] au Nigeria, Shaji et al. [53] en Inde and Rait et al. [49] en Angleterre ont montré que l'histoire familiale de démence était un important facteur de risque de survenue de la démence chez les personnes âgées. En plus, de nombreuses études ont démontré le rôle primordial que joue la présence de l'allèle $\varepsilon 4$ de l'apolipoprotéine $E$ dans la survenue de la maladie d'Alzheimer chez la population caucasienne et afro-américaine [28,44] même si cette association est absente chez certaines populations africaines [19].

La maladie de Parkinson, la deuxième affection neurodégénérative, est aussi fréquente chez la personne âgée et augment avec l'âge [12]. Son association avec la démence a fait l'objet de plusieurs études et sa prévalence varie entre 24,5 et $31,1 \%$ dans la population de patients [1]. Dans notre population à l'étude, la prévalence de la maladie de Parkinson était de $1,5 \%$ et cette affection avait un effet protecteur sur la survenue de démence. Ceci est en contradiction avec les résultats d'études populationnelles qui montrent une prévalence élevée de démence parmi la population de personnes atteintes de la maladie de Parkinson $[12,29]$. Cependant, cet effet protecteur pourrait être lié au traitement dopaminergique reçu par ces patients. En effet, un déficit en dopamine a été observé chez les patients atteints de démence [34]. En plus, la dopaminothérapie a fait l'objet d'essai clinique chez des patients atteints de démence [31]. C'est pourquoi, eu égard à ce résultat, des études plus poussées permettraient de mieux élucider cette hypothèse d'un éventuel rôle protecteur d'une dopaminothérapie chez la personne âgée qui, si elle s'avère positive, ouvrirait la voie de la prévention contre la démence.

La gastrite, avec Helicobacter pylori qui lui est associé, a été incriminée dans la survenue de la maladie d'Alzheimer chez des personnes âgées [35,39]. Dans notre population à l'étude, 22,6\% ont présenté des troubles digestifs (sous forme de gastrite ou de colopathie) dans leurs antécédents avec un effet protecteur sur la survenue de la démence. Qu'est ce qui pourrait expliquer cette association inverse? Est-ce la médication utilisée ou existe-t-il d'autres facteurs explicatifs en rapport avec des problèmes méthodologiques? Néanmoins, cette association mérite des recherches plus poussés pour mieux élucider ce phénomène.

Cette étude est l'une des premières recherches menées sur la démence de la personne âgée au Sénégal. Elle a permis d'identifier des facteurs de risque de démence dans une population de personnes âgées sénégalaises. En plus, elle vient confirmer le rôle de ces facteurs de risque dans la survenue de démence 
comme observé lors d'études réalisées dans le monde développé. Elle comporte cependant des faiblesses en rapport avec sa validité externe et la temporalité des résultats obtenus.

Néanmoins, elle ouvre la voie de la recherche sur cette affection au Sénégal. La population sénégalaise est entrain d'amorcer la transition démographique et il faut planifier pour des services adaptés aux besoins des malades atteints de démence et mettre en œuvre un programme de prévention. Pour cela, il y'a lieu de sensibiliser le personnel de santé, les décideurs politiques ainsi que la communauté sur l'existence de cette affection et de ses facteurs de risque pour une meilleure prévention. 
Tableau I : Caractéristiques sociodémographiques, mode de vie et réseau social de la population à l'étude $(n=872)$.

\begin{tabular}{|l|c|c|}
\hline Variables & $n$ & $\%$ \\
\hline 1- Caractéristiques sociodémographiques & & \\
\hline $\begin{array}{l}\text { Age } \\
\text { Moyenne (Ecart-Type) : 67,2 ans (7,5) }\end{array}$ & & \\
Minimum: 55 ans \\
Maximum : 90 ans & & \\
\hline 55-59 ans & 149 & 17,1 \\
\hline $60-64$ ans & 216 & 24,8 \\
\hline 65-69ans & 160 & 18,3 \\
\hline 70-74 ans & 178 & 20,4 \\
\hline 75-79 ans & 118 & 13,5 \\
\hline 80-84 ans & 42 & 4,8 \\
\hline 85 ans + & 9 & 1,0 \\
\hline Sexe & & \\
\hline Masculin & 546 & 62,6 \\
\hline Situation matrimoniale & & \\
\hline Marié (e) & 689 & 79 \\
\hline Education & & \\
\hline Oui & 430 & 49,3 \\
\hline 2- Habitudes toxiques & & \\
\hline A déjà fumé le tabac (oui) & 224 & 25,7 \\
\hline A déjà consommé de l'alcool (oui) & 77 & 8,8 \\
\hline 3- Activités physiques & & \\
\hline Fait de la marche (oui) & 833 & 95,5 \\
\hline Autres activités physiques & & \\
\hline $\begin{array}{l}\text { Moyenne (Ecart-Type) : 0,2 (0,7) } \\
\text { Minimum: 0 } \\
\text { Maximum : 5 }\end{array}$ & & \\
\hline Absence d'activité & & \\
\hline 1 activité & 738 & 84,6 \\
\hline 2 activités et plus & 83 & 9,5 \\
\hline $\begin{array}{l}\text { 4- Réseau social } \\
\text { Diversité des liens avec les proches } \\
\text { (époux (se), frères/sœurs, amis) } \\
\text { Moyenne (Ecart-Type) : 3,6 (0,6) } \\
\text { Minimum: 0 } \\
\text { Maximum: 4 }\end{array}$ & 51 & 5,8 \\
\hline 0-2 liens & & \\
\hline 3 liens & & \\
\hline 4 liens & & \\
\hline Fréquence hebdomadaire des contacts & \\
\hline
\end{tabular}




\begin{tabular}{|l|c|c|}
\hline Variables & $\boldsymbol{n}$ & $\%$ \\
\hline $\begin{array}{l}\text { avec les proches } \\
\text { (enfants, frères/sœurs, amis) } \\
\text { Moyenne (Ecart-Type) : 5,2 (1,1) } \\
\text { Minimum: 0 } \\
\text { Maximum: } 6\end{array}$ & & \\
\hline $0-3$ contacts & 89 & 10,2 \\
\hline 4 contacts & 139 & 15,9 \\
\hline 5 contacts & 114 & 13,1 \\
\hline 6 contacts & 530 & 60,8 \\
\hline Membre d'association communautaire (oui) & 118 & 13,5 \\
\hline Membre d'association religieuse (oui) & 342 & 39,2 \\
\hline Démence (oui) & 58 & 6,6 \\
\hline
\end{tabular}

\section{Tableau II. Comorbidité}

\begin{tabular}{|l|l|l|}
\hline \multicolumn{1}{|c|}{ Variables } & Fréquence (n) & Pourcentage (\%) \\
\hline Hypertension artérielle (Oui) & 482 & 55,3 \\
\hline Cardiopathie (Oui) & 53 & 6,1 \\
\hline Maladies vasculaires périphériques (Oui) & 42 & 4,8 \\
\hline AVC (Oui) & 42 & 4,8 \\
\hline Diabète (Oui) & 86 & 9,9 \\
\hline Affections respiratoires (Oui) & 126 & 14,4 \\
\hline Affections rhumatismales (Oui) & 410 & 47,0 \\
\hline Cancer/tumeur bénigne (Oui) & 8 & 0,9 \\
\hline Maladie de Parkinson (Oui) & 13 & 1,5 \\
\hline Epilepsie (Oui) & 12 & 1,4 \\
\hline Affections génito-urinaires (Oui) & 119 & 13,6 \\
\hline Cataracte (Oui) & 99 & 11,4 \\
\hline Glaucome (Oui) & 11 & 1,3 \\
\hline Troubles auditifs (Oui) & 68 & 7,8 \\
\hline Troubles digestifs (Oui) & 197 & 22,6 \\
\hline Anémie (Oui) & 90 & 10,3 \\
\hline Affection thyroïdienne (Oui) & 6 & 0,7 \\
\hline Fracture (Oui) & 59 & 6,8 \\
\hline Chute / traumatisme crânien (Oui) & 74 & 8,5 \\
\hline Histoire familiale de démence (Oui) & 69 & 7,9 \\
\hline
\end{tabular}


Tableau III. Prévalence de la démence selon les caractéristiques sociodémographiques, les habitudes toxiques, l'activité physique et le réseau social

\begin{tabular}{|c|c|c|c|}
\hline Variables & $n$ & Prévalence (\%) & P-value \\
\hline 1- Caractéristiques sociodémographiques & & & $0,001^{*}$ \\
\hline \multicolumn{4}{|l|}{ Age } \\
\hline $55-59$ ans & 3 & 2,0 & \\
\hline $60-64$ ans & 10 & 4,6 & \\
\hline 65-69ans & 8 & 5,0 & \\
\hline $70-74$ ans & 16 & 9,0 & \\
\hline $75-79$ ans & 12 & 10,2 & \\
\hline $80-84$ ans & 7 & 16,7 & \\
\hline 85 ans + & 2 & 22,2 & \\
\hline \multicolumn{4}{|l|}{ Sexe } \\
\hline Masculin / Féminin & $39 / 19$ & $7,1 / 5,8$ & 0,45 \\
\hline \multicolumn{4}{|l|}{ Situation matrimoniale } \\
\hline Marié (e) / Non marié (e) & $41 / 17$ & $6,0 / 9,3$ & 0,10 \\
\hline \multicolumn{4}{|l|}{ Education } \\
\hline Oui / Non & $40 / 18$ & $9,3 / 4,1$ & $0,002^{*}$ \\
\hline \multicolumn{4}{|l|}{ 2- Habitudes toxiques } \\
\hline \multicolumn{4}{|l|}{ Avoir fumé } \\
\hline Oui / Non & $13 / 45$ & $5,8 / 6,9$ & 0,55 \\
\hline \multicolumn{4}{|l|}{ Avoir consommé l'alcool } \\
\hline Oui / Non & $4 / 54$ & $5,2 / 6,8$ & 0,59 \\
\hline \multicolumn{4}{|l|}{ 3- Activités physiques } \\
\hline \multicolumn{4}{|l|}{ Marche } \\
\hline Oui / Non & $51 / 7$ & $6,1 / 17,9$ & $0,004^{*}$ \\
\hline \multicolumn{4}{|l|}{ Activités physiques autres que la marche } \\
\hline Pas d'activité & 51 & 6,9 & \\
\hline 1 activité & 3 & 3,6 & 0,49 \\
\hline 2 activités et plus & 4 & 7,8 & \\
\hline \multicolumn{4}{|l|}{ 4- Réseau social } \\
\hline Diversité des liens avec les proches (mari, enfants, frères/sœurs, amis) & & & $0,000^{*}$ \\
\hline $0-3$ liens & 9 & 21,4 & \\
\hline 4 liens & 20 & 8,8 & \\
\hline 5-6 liens & 29 & 4,8 & \\
\hline \multicolumn{4}{|l|}{ Membre d'association communautaire } \\
\hline Oui / Non & $7 / 51$ & $5,9 / 6,8$ & 0,74 \\
\hline \multicolumn{4}{|l|}{ Membre d'association religieuse } \\
\hline Oui / Non & $18 / 40$ & $5,3 / 7,5$ & 0,19 \\
\hline
\end{tabular}




\begin{tabular}{|l|l|l|l|}
\hline \multicolumn{1}{|c|}{ Variables } & $\boldsymbol{n}$ & Prévalence (\%) & $\boldsymbol{P}$-value \\
\hline Fréquence des contacts avec les proches (enfants, frères/sœurs, amis) & & & $\mathbf{0 , 0 0 0 ^ { * }}$ \\
\hline $0-3$ contacts & 15 & 16,9 & \\
\hline 4 contacts & 18 & 12,9 & \\
\hline 5 contacts & 11 & 9,6 & \\
\hline 6 contacts & 14 & 2,6 & \\
\hline
\end{tabular}

Tableau IV. Prévalence de la démence en fonction de la comorbidité

\begin{tabular}{|l|l|l|l|}
\hline Variables & $\mathbf{n}$ & Prévalence & P-value \\
\hline Hypertension artérielle & & & \\
\hline Oui / Non & $41 / 17$ & $8,5 / 4,4$ & $\mathbf{0 , 0 1 5 ^ { * }}$ \\
\hline Cardiopathie & & & \\
\hline Oui / Non & $7 / 51$ & $13,2 / 6,2$ & $\mathbf{0 , 0 4 8 ^ { * }}$ \\
\hline Maladies vasculaires périphériques & & & \\
\hline Oui / Non & $3 / 55$ & $7,1 / 6,6$ & 0,9 \\
\hline AVC & & & \\
\hline Oui / Non & $10 / 48$ & $23,8 / 5,8$ & $\mathbf{0 , 0 0 0}$ \\
\hline Diabète & & & \\
\hline Oui / Non & $2 / 56$ & $2,3 / 7,1$ & 0,09 \\
\hline Affections respiratoires & & & \\
\hline Oui / Non & $6 / 52$ & $4,8 / 7,0$ & 0,36 \\
\hline Affections rhumatismales & & & \\
\hline Oui / Non & $20 / 38$ & $4,9 / 8,2$ & $\mathbf{0 , 0 4 8}$ \\
\hline Cancer/tumeur bénigne & & & \\
\hline Oui / Non & $0 / 58$ & $0,0 / 6,7$ & 0,45 \\
\hline Maladie de Parkinson & & & \\
\hline Oui / Non & $1 / 57$ & $7,7 / 6,6$ & 0,88 \\
\hline Epilepsie & & & \\
\hline Oui / Non & $5 / 53$ & $41,7 / 6,2$ & $\mathbf{0 , 0 0 0}$ \\
\hline Affections génito-urinaires & & & \\
\hline Oui / Non & $12 / 46$ & $10,1 / 6,1$ & 0,10 \\
\hline Cataracte & & & \\
\hline Oui / Non & $7 / 51$ & $7,1 / 6,6$ & 0,86 \\
\hline Glaucome & & & \\
\hline Oui / Non & & & \\
\hline Troubles auditifs & & & \\
\hline Oui / Non & & & \\
\hline Troubles digestifs & & & \\
\hline
\end{tabular}




\begin{tabular}{|l|l|l|l|}
\hline Oui / Non & $6 / 52$ & $3,0 / 7,7$ & $\mathbf{0 , 0 2}^{*}$ \\
\hline Anémie & & & \\
\hline Oui / Non & $9 / 49$ & $10,0 / 6,3$ & 0,18 \\
\hline Affection thyroïdienne & & & \\
\hline Oui / Non & $0 / 58$ & $0,0 / 6,7$ & 0,51 \\
\hline Fractures & & & \\
\hline Oui / Non & $5 / 53$ & $8,5 / 6,5$ & 0,56 \\
\hline Chute / traumatisme crânien & & & \\
\hline Oui / Non & $11 / 47$ & $14,9 / 5,9$ & $\mathbf{0 , 0 0 3}^{*}$ \\
\hline Histoire familiale de démence & & & \\
\hline Oui / Non & $27 / 31$ & $39,1 / 3,9$ & $\mathbf{0 , 0 0 0}^{*}$ \\
\hline
\end{tabular}

Tableau V. Résultats de l'analyse multivariée

\begin{tabular}{|l|l|l|}
\hline \multicolumn{1}{|c|}{ Variables } & Odds ratio & Intervalle de confiance à 95\%) \\
\hline Age & & \\
\hline $55-59$ ans & 1 & - \\
\hline $60-64$ ans & 3,01 & $0,62-14,47$ \\
\hline $65-69$ ans & 2,96 & $0,60-14,59$ \\
\hline $70-74$ ans & 6,53 & $1,42-29,91$ \\
\hline $75-79$ ans & 5,90 & $1,24-28,09$ \\
\hline $80-84$ ans & 11,20 & $2,00-62,70$ \\
\hline 85 ans + & 18,33 & $1,54-217,76$ \\
\hline $\begin{array}{l}\text { Fréquence des contacts hebdomadaires } \\
\text { proches (enfants, frères/sœurs, amis) }\end{array}$ & & \\
\hline $0-3$ contacts & 4,37 & $1,80-10,61$ \\
\hline 4 contacts & 2,55 & $1,06-6,09$ \\
\hline 5 contacts & 2,24 & $0,84-5,94$ \\
\hline 6 contacts & 1 & - \\
\hline AVC & 3,20 & $1,10-9,35$ \\
\hline Maladie de Parkinson & 0,02 & $0,00-0,89$ \\
\hline Epilepsie & 38,42 & $4,86-303,74$ \\
\hline Troubles digestifs & 0,25 & $0,09-0,70$ \\
\hline Histoire familiale de démence & 14,48 & $7,03-29,81$ \\
\hline & & \\
\hline
\end{tabular}




\section{REFERENCES}

1. AARSLAND D, ANDERSEN K, LARSEN JP, LOLK A, KRAG-SORENSEN P. Prevalence and characteristics of dementia in Parkinson disease. An 8-year prospective study. Arch Neurol 2003; 60:387-92.

2. ALVARADO BE, ZUNZUNEGUI MV, DEL SER T, BELAND F. Cognitive decline is related to education and occupation in a Spanish elderly cohort. Aging (Milano) 2002; 14 (2):132-2.

3. AMERICAN PSYCHIATRIC ASSOCIATION. Diagnostic and Statistical Manual of Mental Disorders, 4th Ed. Am Psychiatric Assoc, 1994; Washington DC.

4. ANNEGERS JF, HAUSER WA, LEE J, ROCCA W. Incidence of acute symptomatic seizures in Rochester, Minnesota, 1935-1984. Epilepsia 1995; 36:327-33.

5. ATTI AR, PALMER K, VOLPATO S, ZULIANI G, WINBLAD B, FRATIGLIONI L. Anaemia increases the risk of dementia in cognitively intact elderly. Neurobiol Aging 2006; 27:278-284.

6. BAIYEWU O, BELLA AF, JEGEDE O. The effect of demographic and health variables on a modified form of Mini Mental State Examination scores in Nigerian elderly community residents. Int J Geriatr Psychiatry 1993; 8:503-510.

7. BASSUK SS, GLASS TA, BERKMAN LF. Social disengagement and incident cognitive decline in community-dwelling elderly persons. Ann Intern Med 1999; 131:165-73.

8. BELAND F, ZUNZUNEGUI MV, ALVARADO B, OTERO A, DEL SER T. Trajectories of cognitive decline and social relations. J Gerontol Psychol Sci 2005; 60B (6):320-330.

9. BENNETT DA, SCHNEIDER JA, TANG Y, ARNOLD SE, WILSON RS. The effect of social networks on the relation between Alzheimer's disease pathology and level of cognitive function in old people: a longitudinal cohort study. Lancet Neurol 2006; 5:406-412.

10.BORENSTEIN AR, COPENHAVER CI, MORTIMER JA. Early-life risk factors for Alzheimer disease. Alz Dis Assoc Disord 2006; 20 (1):63-72.

11.BRETELER MMB, de GROOT RR, van ROMUNDE LK, HOFMAN A. Risk of dementia in patients with Parkinson's disease, epilepsy and severe head trauma: a register-based follow-up. Am J Epidemiol 1995; 142 (12):1300-5.

12.De LAU LML, SCHIPPER CMA, HOFMAN A, KOUDSTALL PJ, BRETELER MMB. Prognosis of Parkinson disease. Risk of dementia and mortality: The Rotterdam Study. Arch Neurol 2005; 62:1265-9.

13.DENG J, ZHOU DHD, LI J, WANG J, GAO C, CHEN M. A 2-year follow-up study of alcohol consumption and risk of dementia. Clin Neurol Neurosurg 2006; 108:378-383.

14.EVANS DA, FUNKENSTEIN HH, ALBERT MS, et al. Prevalence of Alzheimer's disease in a community population of older persons: higher than previously reported. JAMA 1989; 262:25512556.

15.FILLENBAUM GG. Screening the Elderly. A Brief Instrumental Activities of Daily Living Measure. J Am Geriatr Soc 1985; 33:698-706.

16.FOLSTEIN MF, FOLSTEIN SE, McHUGH PR. 'Mini Mental State': a practical method for grading the cognitive state of patients for the clinician. Psych Med 1975; 12:189-198.

17.FRATIGLIONI L, PAILLARD-BORG S, WINBLAD B. An active and socially integrated lifestyle in late life might protect against dementia. Lancet Neurol 2004; 3:343-53

18.GRIFFITH HR, MARTIN RC, BAMBARA JK, MARSON DC, FAUGHT E. Older adults with epilepsy demonstrate cognitive impairments compared with patients with amnestic mild cognitive impairment. Epilepsy \& Behav 2006; 8:161-8.

19. GUREJE O, OGUNNIYI A, BAIYEWU O, et al. APOE $\varepsilon 4$ is not associated with Alzheimer's disease in elderly Nigerians. Ann Neurol 2006; 59:182-5.

20.HALL K, GUREJE O, GAO S et al. Risk factors and Alzheimer's disease: a comparative study of two communities. Aust N Z J Psychiatry 1998; 32 (5):698-706.

21. HAUSER WA, ANNEGERS JF, KURLAND LT. Prevalence of epilepsy in Rochester, Minnesota: 1940-1980. Epilepsia 1991; 32:429-45.

22. HAYDEN KM, ZANDI PP, LYKETSOS CG, et al.. Vascular risk factors for incident Alzheimer disease and vascular dementia: The Cache County Study. Alz Dis Assoc Disord 2006; 20 (2):93100.

23. HERRERA E, CARAMELLI P, SILVEIRA ASB, NITRINI R. Epidemiologic survey of dementia in a community-dwelling Brazilian population. Alz Dis Assoc Disord 2002; 16(2):103-108.

24.HO SC, WOO J, SHAM A, CHAN SG, YU ALM. A 3-year follow-up study of social, lifestyle and health predictors of cognitive impairment in a Chinese older cohort. Int J Epidemiol 2001; 30:1389- 
1396.

25. HODKINSON HM. Evaluation of a mental test score for assessment of mental impairment in the elderly. Age Ageing 1972; 1:233-238.

26.http.www.finances.gouv.sn, 2005)

27.http.www.un.org, 2002)

28. HUANG W, QIU C, von STRAUSS E, WINBLAD B, FRATIGLIONI L. APOE genotype, family history of dementia and Alzheimer disease risk: a 6 year follow-up study. Arch Neurol 2004; 61:1930-1934.

29.INCALZI RA, MARRA C, GIORDANO A, et al.. Cognitive impairment in chronic obstructive pulmonary disease. A neuropsychological and SPECT study. J Neurol 2003; 250:325-332.

30.IVAN CS, SESHADRI S, BEISER A et al. Dementia after stroke: The Framingham Study. Stroke 2004; 35:1264-69.

31.JELLINGER K, FLAMENT H, RIEDERER P, SCHMID H, AMBROZI L. Levodopa in the treatment of (pre) senile dementia. Mech Ageing Dev 1980; 14 (1-2):253-64.

32.JITAPUNKUL S, PILLAY I, EBRAHIM S. The Abbreviated Mental Test: its use and validity. Age Ageing 1991; 20:332-336.

33.KAHANA E, GALPER Y, ZILBER N, KORCZYN AD. Epidemiology of dementia in Ashkelon. J Neurol, 2003; 250:424-428.

34.KEMPPAINEN N, LAINE M, LAAKSO MP, et al. Hippocampal dopamine D2 receptors correlate with memory functions in Alzheimer's disease. Eur J Neurosci 2003; 18 (1):149-54.

35.KOUNTOURAS J, TSOLAKI M, GAVALAS E, et al. Relationship between Helicobacter pylori infection and Alzheimer disease. Neurology 2006; 66 (6):938-40.

36. LAUNER L, ANDERSEN K, DEWEY ME, et al.. Rates and risk factors for dementia and Alzheimer's disease: results from EURODEM pooled analyses. Neurology 1999; 52 (1):78-84.

37.LAWTON MP, BRODY EM. Assessment of older people: self-maintaining and instrumental activities of daily living. Gerontol 1969; 9:179-186.

38.LEYS D, Hénon H, Mackowiack-Cordoliani M, Pasquier F. Poststroke dementia. Lancet Neurol 2005; 4:752-9

39.MALAGUARNERA M, BELLA R, ALAGONA G, FERRI R, CARNEMOLLA A, PENNISI G. Helicobacter pylori and Alzheimer's disease: a possible link. Eur J Int Med 2004; 15:381-6.

40.MARTIN RC, GRIFFITH HR, FAUGHT E, GILLIAM F, MACKEY ML, VOGTLE LK. Cognitive functioning in community-dwelling older adults with chronic partial epilepsy. Epilepsia 2005; 46:298303.

41.MCEWEN BS. Sex, stress and the hippocampus: allostasis, allostatic and the aging process. Neurobiol Aging 2002; 23:921-939.

42.MEGURO K, ISHII H, YAMAGUCHI S, et al.. Prevalence of dementia and dementing diseases in Japan. The Tajiri Project. Arch Neurol 2002; 59:1109-1114.

43.MRC Cognitive Function and Ageing Study, YIP AG, BRAYNE C, MATHEWS FE. Risk factors for incident dementia in England and Wales: The Medical Research Council Cognitive Function and Ageing Study: a population-based nested case-control study. Age Ageing 2006; 35:154-60.

44.MURRELL JR, PRICE B, LANE KA, et al. Association of Apolipoprotein E genotype and Alzheimer disease in African Americans. Arch Neurol 2006; 63:431-5

45.NDIAYE MM, SENE F, NDIAYE IP. Les accidents vasculaires cérébraux. Forum Méd.1994; 4 : 3-4.

46. OTT A, SLOTER AJC, HOFMAN A, et al.. Smoking and risk of dementia and Alzheimer's disease in a population-based cohort study: The Rotterdam Study. Lancet 1998; 351:1840-1843.

47.PODEWILS LJ, GUALLAR E, KULLER LH, et al. Physical activity, ApoE genotype and dementia risk: findings from the Cardiovascular Health Cognition Study. Am J Epidemiol 2005; 161 (7):63951.

48.RADLOFF LS. The CES-D scale: a self report depression scale for research in the general population. Appl Psychol Meas 1977; 1:385-401.

49. RAIT G, FLETCHER A, SMEETH L, et al. Prevalence of cognitive impairment: results from the MRC trial of assessment and management of older people in the community. Age Ageing 2005; 34:242-8.

50.RAJKUMAR S, KUMAR S, THARA R. Prevalence of dementia in a rural setting: a report from India. Int J Geriatr Psychiatry 1997; 12:702-707.

51.ROSSO SM, LANDWEER EJ, HOUTERMAN M, KAAT LD, VAN DUIJIN CM, VAN SWIETEN LC. Medical and environmental risk factors for sporadic frontotemporal dementia: a retrospective casecontrol study. J Neurol Neurosurg Psychiatry 2003; 74:1574-6.

52.SACZINSKI JS, PFEIFFER LA, MASAKI K, et al. The effect of social engagement on incident dementia: The Honolulu-Asia Aging Study. Am J Epidemiol 2006; 163 (5):433-40.

53.SHAJI S, BOSE S, VERGHESE A. Prevalence of dementia in an urban population in Kerala, India. 
Br J Psychiatry 2005; 186:136-40.

54.STERN Y. Cognitive reserve and Alzheimer disease. Alz Dis Assoc Disord 2006; 20 (2):112-117.

55.TILVIS RS, KÄHÖNEN-VÄRE MH, JOLKKONEN J, VALVANNE J, PITKALA KH, STRANDBERG TE. Predictors of cognitive decline and mortality of aged people over a 10 year period. J Gerontol Med Sci 2004; 59A (3):268-274.

56.TOURÉ K. Développement et validation d'un test de dépistage de la démence chez une population de personnes âgées sénégalaises: le Test du Sénégal. Thèse de Doctorat en Santé Publique option Epidémiologie, Département de Médecine Sociale et Préventive, Faculté de Médecine, Université de Montréal, Québec-Canada, Octobre 2007:p261.

57.VAN PRAAG H, KEMPERMANN G, GAGE FH. Neural consequences of environmental enrichment. Nat Rev Neurosci 2000; 1:191-198.

58.WANG H, KARP A, WINBLAD B, FRATIGLIONI L. Late-life engagement in social and leisure activities is associated with a decreased risk of dementia: a longitudinal study from the Kungsholmen Project. Am J Epidemiol 2002; 155 (12):1081-7.

59.WHO. World health Report. 2000. Geneva, Switzerland.

60.WIMO A, WINBLAD B, AGUERO-TORRES H, VON STRAUSS E. The magnitude of dementia occurrence in the world. Alzheimer Dis Ass Disord 2003 17(2):63-67.

61.WOO JI, LEE JH, YOO K, KIM C, KIM Y, SHIN YS. Prevalence estimation of dementia in a rural area of Korea. J Am Geriatr Soc 1998; 46:983-987.

62.ZHU L, FRATIGLIONI L, GUO Z, AGÜERO-TORRES H, WINBLAD B, VIITANEN M. Association of stroke with dementia, cognitive impairment and functional disability in the very old. A populationbased study. Stroke 1998; 29:2094-9.

63.ZUNZUNEGUI MV, ALVARADO BE, DEL SER T, OTERO A. Social networks, social integration and social engagement determine cognitive functions in community-dwelling Spanish older adults. J Gerontol B Psychol Soc Sci 2003; 58(2):S93-S100. 